PROCEEDINGS OF THE

AMERICAN MATHEMATICAL SOCIETY

Volume 134, Number 8 , Pages $2427-2435$

S 0002-9939(06)08189-5

Article electronically published on January 31, 2006

\title{
UNIVERSALITY OF UNIFORM EBERLEIN COMPACTA
}

\author{
MIRNA DŽAMONJA
}

(Communicated by Alan Dow)

\begin{abstract}
We prove that if $\mu^{+}<\lambda=\operatorname{cf}(\lambda)<\mu^{\aleph_{0}}$ for some regular $\mu>2^{\aleph_{0}}$, then there is no family of less than $\mu^{\aleph_{0}}$ c-algebras of size $\lambda$ which are jointly universal for c-algebras of size $\lambda$. On the other hand, it is consistent to have a cardinal $\lambda \geq \aleph_{1}$ as large as desired and satisfying $\lambda^{<\lambda}=\lambda$ and $2^{\lambda^{+}}>\lambda^{++}$, while there are $\lambda^{++}$c-algebras of size $\lambda^{+}$that are jointly universal for calgebras of size $\lambda^{+}$. Consequently, by the known results of M. Bell, it is consistent that there is $\lambda$ as in the last statement and $\lambda^{++}$uniform Eberlein compacta of weight $\lambda^{+}$such that at least one among them maps onto any Eberlein compact of weight $\lambda^{+}$(we call such a family universal). The only positive universality results for Eberlein compacta known previously required the relevant instance of $G C H$ to hold. These results complete the answer to a question of Y. Benyamini, M. E. Rudin and M. Wage from 1977 who asked if there always was a universal uniform Eberlein compact of a given weight.
\end{abstract}

\section{INTRODUCTION}

We present several results connected to the problem of the existence of universal objects in the class of uniform Eberlein compacta and certain Boolean algebras closely connected with them, namely c-algebras. A uniform Eberlein compact, abbreviated UEC, is a topological space homeomorphic to a weakly compact subspace of a Hilbert space. A UEC of weight $\lambda$ is said to be universal of weight $\lambda$ iff every UEC of weight $\lambda$ is a continuous image of it. The question of the existence of universal uniform Eberlein compacta of a given weight was raised by Yoav Benyamini, Mary Ellen Rudin and Michael Wage in their 1977 paper YRM77. Murray Bell in Bel00. proved that there is a universal UEC of weight $\lambda$ iff there is a universal c-algebra of size $\lambda$. (The universal c-algebra is understood to be one into which all others of the same size embed, as Boolean algebras.) In the same paper Bell showed that if $2^{<\lambda}=\lambda$, there is a c-algebra of size $\lambda$ which is universal not just under ordinary embeddings, but also under a stronger notion of a c-embedding. He also provided negative consistency results in models obtained by adding Cohen subsets to a regular cardinal. Mirna Džamonja in Dža98 showed that for no regular cardinal $\lambda>\aleph_{1}$ with $2^{\aleph_{0}}>\lambda$ can there exist a c-algebra of size $\lambda$ into which every c-algebra of size $\lambda$ c-embeds. In fact, under these circumstances, for no $\mu<2^{\aleph_{0}}$ are

Received by the editors February 27, 2002 and, in revised form, February 23, 2005.

2000 Mathematics Subject Classification. Primary 03E35, 03E75, 03C55, 54C35, 46E99.

Key words and phrases. Universal models, c-algebras, Eberlein compacta.

The author thanks EPSRC for their support through the grant number GR/M71121 and the EPSRC Advanced Fellowship, and the referees for their comments on the paper. 
there $\mu$ c-algebras of size $\lambda$ such that every c-algebra of size $\lambda$ embeds into one of the $\mu$ given ones.

This paper contains both negative and positive results about the existence of universal c-algebras and UEC. On the one hand we extend the result of [Dža98] to include more cardinals, and show that the negative result holds even when the embeddings are assumed just to preserve " $\bigcup_{n<\omega} B_{n}$ ", a notion which is weaker than being a c-embedding (see Definition 1.2 below). On the other hand, we prove a positive consistency result showing that under certain non-GCH assumptions there can be a family of UEC of a relatively small size $\left(\lambda^{++}<2^{\lambda^{+}}\right)$each of which has weight $\lambda^{+}$and which are jointly universal for UEC of weight $\lambda^{+}$. The method of the proof of the negative result is an elaboration of the method of invariants used in Dža98. The positive result is proved using an axiom scheme that was proved consistent with $Z F C$ in [DžSh04]. Checking that these axioms apply is non-trivial, and the main point of the proof is to define an auxiliary ordered family, in the proof referred to as the $\lambda$-approximation family, that satisfies a certain (unfortunately rather long) list of requirements. All background necessary for the proofs is contained in $\$ 1$. The negative results are contained in $₫ 2$, and the positive ones in §.

\section{BACKGROUND}

Notation 1.1. For $\alpha>\theta=\operatorname{cf}(\theta)$, we let $S_{\theta}^{\alpha} \stackrel{\text { def }}{=}\{\beta<\alpha: \operatorname{cf}(\beta)=\theta\}$. Throughout $\lambda$ stands for an infinite cardinal. If $\mathcal{K}$ is a class of models, then $\mathcal{K}_{\lambda}$ denotes all elements of $\mathcal{K}$ of size $\lambda$.

Definition 1.2. (1) A subset $C$ of a Boolean algebra $B$ has the nice property if for no finite $F \subseteq C$ do we have $\bigvee F=1$. A Boolean algebra $B$ is a c-algebra iff there is a family $\left\{B_{n}: n<\omega\right\}$ of pairwise disjoint subsets of $B$ each consisting of pairwise disjoint elements, whose union has the nice property and generates $B$. In such a case we say that $\left\langle B_{n}: n<\omega\right\rangle$ witnesses that $B$ is a c-algebra. When discussing c-algebras, we always have in mind a fixed sequence witnessing this, although we may omit to mention it. We may refer to it as $\left\langle B_{n}(B): n<\omega\right\rangle$.

(2) If $B_{l}^{*}$ for $l \in\{0,1\}$ are c-algebras, then a 1-1 Boolean homomorphism $f$ : $B_{0}^{*} \rightarrow B_{1}^{*}$ is a c-embedding iff $f$ " $B_{n}\left(B_{0}^{*}\right) \subseteq B_{n}\left(B_{1}^{*}\right)$ for all $n<\omega$.

(3) A c-algebra $B^{*}$ of size $\lambda$ is c-universal of size $\lambda$ iff for any c-algebra $B$ of size $\lambda$ (equivalently, $\leq \lambda$ ), there is a c-embedding $f: B \rightarrow B^{*}$.

Definition 1.3. (1) Suppose that $B$ is a Boolean algebra of size $\lambda$. An increasing continuous sequence $\bar{B}=\left\langle B^{\alpha}: \alpha<\lambda\right\rangle$ of subalgebras of $B$ is a filtration of $B$ iff $B_{0}=\emptyset,\left|B^{\alpha}\right|<\lambda$ for all $\alpha$, and $\bigcup_{\alpha<\lambda} B^{\alpha}=B$.

(2) Let $B$ be a c-algebra of size $\lambda$ with a filtration $\bar{B}$, and $\left\langle C_{\delta}: \delta \in S^{*} \subseteq \lambda\right\rangle$ a sequence of sets with $C_{\delta} \subseteq \delta$. For $\delta \in S^{*}$ and $b \in B \backslash B^{\delta}$ we define

$$
\operatorname{Inv}_{\bar{B}}\left(b, C_{\delta}\right) \stackrel{\text { def }}{=}\left\{\alpha \in C_{\delta}:(\exists m \geq 1)\left(\exists y \in B_{m}(B) \cap B^{\min \left(C_{\delta} \backslash(\alpha+1)\right)} \backslash B^{\alpha}\right)[y \geq b]\right\} .
$$

If $\alpha \in \operatorname{Inv}_{\bar{B}}\left(b, C_{\delta}\right)$ because $m \geq 1$ is such that for some $y \geq b$ we have $y \in$ $B_{m}(B) \cap B^{\min \left(C_{\delta} \backslash(\alpha+1)\right)} \backslash B^{\alpha}$, we say that $\alpha \in \operatorname{Inv}_{\bar{B}}\left(b, C_{\delta}\right)$ by virtue of $m$ (note that there can be at most one such $\alpha$ for $b \neq 0$ ).

By a slight modification of the proof of Main Claim 12 in Dža98, as indicated, we obtain the following theorem. 
Theorem 1.4. Suppose that $S^{*} \subseteq \lambda$ and $\bar{C}=\left\langle C_{\delta}: \delta \in S^{*}\right\rangle$ is a sequence with $C_{\delta} \subseteq \delta$, while $\bar{A}=\left\langle A_{\delta}: \delta \in S^{*}\right\rangle$ is a sequence of distinct sets with $\operatorname{otp}\left(A_{\delta}\right)=\omega$ and $A_{\delta} \subseteq C_{\delta}$.

Then there is a c-algebra $B=B[\bar{A}]$ and filtration $\bar{B}=\bar{B}[\bar{A}]$ of $B$ such that for all $\delta \in S^{*}$ there is $b_{\delta}^{0} \in B \backslash B^{\delta}$ such that $\operatorname{Inv}_{\bar{B}}\left(b_{\delta}^{0}, C_{\delta}\right)=A_{\delta}$.

Sketch of the proof. The proof in Dža98, is formulated for the situation relevant there, namely when $\bar{C}$ is a club-guessing sequence and $\operatorname{otp}\left(C_{\delta}\right)=\omega$ while $\sup (\delta)=$ $\delta$. However these facts are only used to conclude that the increasing enumerations of $A_{\delta}$ 's are pairwise eventually different. This is anyway true in the present situation.

Theorem 1.5 (Bell, Bel00). For every two cardinals $\lambda \geq \aleph_{0}$ and $\mu$, there are $\mu$ c-algebras of size $\lambda$ that are jointly universal for c-algebras of size $\lambda$ iff there are $\mu$ UEC of weight $\lambda$ that are jointly universal for UEC of weight $\lambda$.

We recall some definitions from DžSh04, given in the simpler form appropriate to what we need here. The universe of a model $M$ in the following is denoted by $|M|$.

Definition 1.6. A pair $K_{\mathrm{ap}}=\left(K_{\mathrm{ap}}, \leq_{\mathrm{ap}}\right)$ is a (strong) $\lambda$-approximation family iff for some finitary vocabulary $\tau$ of size $\leq \lambda$ the following axioms (a)-(i) hold:

(a)-(c) $K_{\text {ap }}$ is a set of $\tau$-models partially ordered by $\leq_{\text {ap }} 11$ If $M \in K_{\text {ap }}$, then $|M| \in\left[\lambda^{+}\right]^{<\lambda}$ and $M \leq_{\text {ap }} N \Longrightarrow M \subseteq N$. If $M \in K_{\text {ap }}$ and $\delta$ is divisible by $\lambda$ (which we write as $\lambda \mid \delta$ ), then $M \uparrow \delta \in K_{\text {ap }}$ and $M \uparrow \delta \leq_{\text {ap }} M$. If $M, N \in K_{\text {ap }}$ and $\lambda \mid \delta$, while $M \leq_{\text {ap }} N$, then $M \uparrow \delta \leq_{\text {ap }} N \mid \delta$.

(d) [Union] Suppose that $i^{*}<\lambda$. If $\bar{M}=\left\langle M_{i}: i<i^{*}\right\rangle$ is a $\leq_{\text {ap-increasing }}$ sequence in $K_{\text {ap }}$, then $\bigcup_{i<i^{*}} M_{i}$ is an element of $K_{\text {ap }}$, and it is the $\leq_{\text {ap-lub }}$ (least upper bound) of $\bar{M}$.

(e) [End extension/Amalgamation] If $0<\delta<\lambda^{+}$is divisible by $\lambda$, and $M_{0}, M_{1}$, $M_{2} \in K_{\text {ap }}$ are such that $M_{2}\left\lceil\delta=M_{0} \leq_{\text {ap }} M_{1}\right.$ and $\left|M_{1}\right| \subseteq \delta$, then

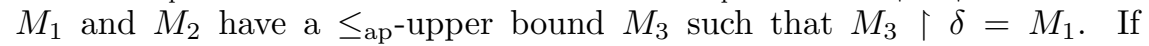
$M_{0}, M_{1}, M_{2}, \delta$ are as above and $M_{1}, M_{2} \leq M$, then there is $M_{3} \leq M$ such that $M_{3} \geq M_{1}, M_{2}$ and $M_{3}\left\lceil\delta_{1}=M_{1}\right.$.

(f) [Local Cardinality] $\left|\left\{M \in K_{\text {ap }}:|M| \subseteq \alpha\right\}\right| \leq \lambda$ holds for all $\alpha<\lambda^{+}$.

Let $u_{1}, u_{2} \subseteq \lambda^{+}$. A function $h: u_{1} \rightarrow u_{2}$ is said to be lawful iff it is 1-1 onto and for all $\alpha \in u_{1}$ we have $h(\alpha)+\lambda=\alpha+\lambda$. With $K_{\text {ap }}$ as above, a function $h$ is said

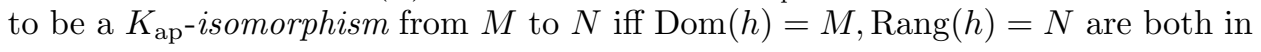
$K_{\text {ap }}$, and $h$ is a $\tau$-isomorphism.

(g) [Uniformity] If $M \in K_{\text {ap }}$ and $h$ is a lawful function from $|M|$ onto some $u \subseteq \lambda^{+}$, then for some $M^{\prime} \in K_{\text {ap }}$ we have that $\left|M^{\prime}\right|=u$ and $h$ is a

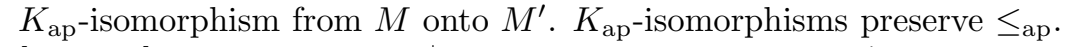

(h) [Density] For every $\beta$ in $\lambda^{+}$and $M \in K_{\text {ap }}$, there is $M^{\prime} \in K_{\text {ap }}$ such that $M \leq_{\text {ap }} M^{\prime}$ and $\beta \in\left|M^{\prime}\right|$.

(i) [Amalgamation] Assume $M_{l} \in K_{\text {ap }}$ for $l<3$ and $M_{0} \leq$ ap $M_{l}$ for $l=1,2$. Then for some lawful function $f$ and $M \in K_{\text {ap }}$, we have $M_{1} \leq_{\text {ap }} M$, the domain of $f$ is $M_{2}$, the restriction $f \uparrow\left|M_{0}\right|$ is the identity, and $f$ is a $\leq_{\text {ap }^{-}}$ embedding of $M_{2}$ into $M$, i.e. $f\left(M_{2}\right) \leq$ ap $M$. If $M_{1} \cap M_{2}=M_{0}$, we can assume that $f=$ id.

\footnotetext{
${ }^{1}$ For the purposes of this definition $\emptyset$ is a $\tau$-model.
} 
Definition 1.7. Let $\left(K_{\mathrm{ap}}, \leq_{\mathrm{ap}}\right)$ be a $\lambda$-approximation family. A subset $\Gamma$ of $K_{\text {ap }}$ is $(<\lambda)$-closed iff for every $\leq_{\mathrm{ap}}$-increasing chain of size $<\lambda$ of elements of $\Gamma$, the lub of the chain is in $\Gamma$. We let $K_{\mathrm{md}}^{-}$be the set of all $(<\lambda)$-closed $\Gamma$ which are $\leq_{\mathrm{ap}^{-}}$ directed and such that for cofinally many $\beta<\lambda^{+}$there are $M \in \Gamma$ and $\gamma \in|M|$ such that $\min \{\delta: \gamma<\delta \& \lambda \mid \delta\}=\min \{\delta: \beta<\delta \& \lambda \mid \delta\}$. We let $K_{\text {md }}$ contain those $\Gamma \in K_{\text {md }}^{-}$for which $\left[M \in \Gamma \& M \leq_{\text {ap }} M_{1} \Longrightarrow\left(\exists M_{2} \in \Gamma\right)(\exists h\right.$ lawful $)\left[h: M_{1}\right.$ $\rightarrow M_{2}$ embedding over $\left.M\right]$, and which are downwards closed with respect to $\leq_{\text {ap }}$.

Definition 1.8. For a $\lambda$-approximation family $K_{\text {ap }}$ we say that it is workable iff for every $\Gamma \in K_{\mathrm{md}}^{-}$such that $M \in \Gamma \Longrightarrow|M| \subseteq$ the class Ev of even ordinals, for all $\delta_{1}<\delta_{2} \in S_{\lambda}^{\lambda^{+}}$the following holds:

Suppose that for $l \in\{1,2\}$ we have $\left(M_{l}, N_{l}\right)$ such that $M_{l} \in \Gamma, M_{l} \leq_{\text {ap }} N_{l}$, and $\left|N_{l}\right| \cap \mathrm{Ev}=\left|M_{l}\right|$. In addition suppose that $\left|N_{1}\right| \subseteq \delta_{2}, N_{1}\left\lceil\delta_{1}=N_{2}\left\lceil\delta_{2}\right.\right.$ and that some $h$ is a $K_{\text {ap }}$-isomorphism from $N_{1}$ onto $N_{2}$ mapping $M_{1}$ onto $M_{2}$, while $h \uparrow\left(N_{1}\left\lceil\delta_{1}\right)\right.$ is the identity. Then there are $M \in \Gamma$ and $N \in K_{\text {ap }}$ and $g_{l}$ for $l \in\{1,2\}$ such that $M_{l} \leq_{\text {ap }} M \leq_{\text {ap }} N$ and $g_{l}$ is a $\leq_{\text {ap }}$-embedding of $N_{l}$ into $N$, with $g_{l}\left\lceil M_{l}=\operatorname{id}_{M_{l}}\right.$. In addition, $|N| \cap \mathrm{Ev}=|M|$ and $g_{l} \uparrow\left(N_{l} \uparrow \delta_{l}\right)$ is fixed.

Definition 1.9. $\mathcal{K}=\left(K, \leq_{\mathcal{K}}\right)$ is an abstract elementary class iff $K$ is a class of models of some vocabulary $\tau_{\mathcal{K}}=\tau$ and $\leq_{\mathcal{K}}$ is a partial order on $K$, satisfying:

(i) If $M \in K$, then all $\tau$-models isomorphic to $M$ are also in $K$. The relation $\leq_{\mathcal{K}}$ is preserved under isomorphisms.

(ii) If $M \leq_{\mathcal{K}} N$, then $M$ is a submodel of $N$.

(iii) The union of a $\leq_{\mathcal{K}}$-increasing continuous chain $\bar{M}$ of elements of $K$ is an element of $K$ and the lub of $\bar{M}$ under $\leq_{\mathcal{K}}$.

(iv) If $M_{l} \leq_{\mathcal{K}} N$ for $l \in\{0,1\}$ and $M_{0}$ is a submodel of $M_{1}$, then $M_{0} \leq_{\mathcal{K}} M_{1}$.

(v) There is a cardinal $\kappa$ such that for every $M \in \mathcal{K}$ and $A \subseteq|M|$, there is $N \leq \mathcal{K} M$ such that $A \subseteq|N|$ and $\|N\| \leq \kappa \cdot(|A|+1)$. The least such $\kappa$ is denoted by $\operatorname{LS}(\mathcal{K})$ and called the Löwenheim-Skolem number of $\mathcal{K}$.

For such $\mathcal{K}$ we say that it has the joint embedding property JEP iff for any $M_{1}, M_{2} \in \mathcal{K}$, there is $N \in \mathcal{K}$ such that $M_{1}, M_{2}$ are $\leq_{\mathcal{K}}$-embeddable into $N$. $\mathcal{K}$ is said to have amalgamation $A P$ iff for all $M_{0}, M_{1}, M_{2} \in \mathcal{K}$ and $\leq_{\mathcal{K}}$-embeddings $g_{l}: M_{0} \rightarrow M_{l}$ for $l \in\{1,2\}$, there is $N \in \mathcal{K}$ and $\leq_{\mathcal{K}}$-embeddings $f_{l}: M_{l} \rightarrow N$ such that $f_{1} \circ g_{1}=f_{2} \circ g_{2}$.

Definition 1.10. Suppose that $\mathcal{K}$ is an abstract elementary class with $\tau_{\mathcal{K}}=\tau$, and $K_{\text {ap }}$ is a $\lambda$-approximation family whose underlying vocabulary is $\tau$ and such that $K_{\text {ap }} \subseteq \mathcal{K}$ and $\leq_{\text {ap }} \subseteq \leq_{\mathcal{K}}$. In addition suppose that for every $M \in \mathcal{K}$ of size $<\lambda$ there is $N \in K_{\text {ap }}$ with $M \leq_{\mathcal{K}} N$. We say that $K_{\text {ap }}$ tends to $\lambda$-approximate $\mathcal{K}$. $K_{\text {ap }}$ $\lambda$-approximates $\mathcal{K}$ if in addition

(*) For every $M^{*} \in \mathcal{K}_{\lambda^{+}}$, there is $\Gamma \in K_{\text {md }}^{-}\left[K_{\text {ap }}\right]$ with $\{|M|: M \in \Gamma\}$ a club of $[\mathrm{Ev}]^{<\lambda}$ such that for some $M^{\prime}$ isomorphic to $M^{*}$, we have $M^{\prime} \leq_{\mathcal{K}} M_{\Gamma} \stackrel{\text { def }}{=}$ $\bigcup_{M \in \Gamma} M$.

\section{Non-EXistence of Universal C-ALGeBras}

Theorem 2.1. Suppose that $\mu^{+}<\lambda=\operatorname{cf}(\lambda)<\mu^{\aleph_{0}}$ for some $\mu$ regular satisfying $2^{\aleph_{0}}<\mu$. Then:

(1) There is no c-universal c-algebra of size $\lambda$; moreover, no $\left\{B_{i}^{*}: i<i^{*}<\mu^{\aleph_{0}}\right\}$ $c$-algebras of size $\lambda$ are c-universal. 
(2) Moreover, no $\left\{B_{i}^{*}: i<i^{*}<\mu^{\aleph_{0}}\right\}$ c-algebras of size $\lambda$ are universal for c-algebras of size $\lambda$ under embeddings of Boolean algebras which simply preserve " $\in \bigcup_{n<\omega} B_{n} "$.

Proof. We prove (2), which clearly implies (1). By the assumption $\mu^{+}<\lambda$, fix a sequence $\bar{C}=\left\langle C_{\delta}: \delta \in S^{*}\right\rangle$, for some $S^{*} \subseteq \lambda$ stationary, such that $C_{\delta}$ is a closed subset of $\delta$ with $\operatorname{otp}\left(C_{\delta}\right)=\mu$, and such that for every club $E$ of $\lambda$ there are stationary many $\delta$ such that $C_{\delta} \subseteq E$ (the existence of such a sequence was proved by S. Shelah in She94]).

Suppose we are given $\left\{B_{i}^{*}: i<i^{*}<\mu^{\aleph_{0}}\right\}$, c-algebras of size $\lambda$, and for each $i$ a filtration $\bar{B}_{i}^{*}$ of $B_{i}^{*}$. For $\delta \in S^{*}$ we shall choose $A_{\delta} \subseteq C_{\delta}$ with $\operatorname{otp}\left(A_{\delta}\right)=\omega$, and such that for no $x \in \bigcup_{i<i^{*}} B_{i}^{*}$ do we have that $A_{\delta} \cap \operatorname{Inv}_{\bar{B}_{i}^{*}}\left(x, C_{\delta}\right)$ is infinite and $\delta_{1} \neq \delta_{2} \Longrightarrow A_{\delta_{1}} \neq A_{\delta_{2}}$.

To see that such a choice is possible we shall use the well-known fact that there are $\mu^{\aleph_{0}}$ almost disjoint subsets of $\mu$, each of order type $\omega$. The following short proof of this fact was kindly provided by the referee: namely first notice that it is sufficient to find an almost disjoint family in $[\mu]^{\aleph_{0}}$ of size $\mu$; then by taking the first $\omega$ elements of each set in the family we shall have a family as we require. Now for each $x \in[\mu]^{\aleph_{0}}$ let $\varphi_{x}: \omega \rightarrow x$ be a bijection, and let $\rho:{ }^{\omega>} \mu \rightarrow \mu$ be a bijection. Define $\psi:[\mu]^{\aleph_{0}} \rightarrow[\mu]^{\aleph_{0}}$ by letting $\psi(x)=\left\{\rho\left(\left\langle\varphi_{x}(0), \ldots, \varphi_{x}(n)\right\rangle\right): n<\omega\right\}$ and note that the sets in the image of $\psi$ are pairwise almost disjoint.

Hence for $\delta \in S^{*}$ we can find a family $\mathcal{X}_{\delta}$ of $\mu^{\aleph_{0}}$ almost disjoint subsets of $C_{\delta}$, each of order type $\omega$. Now we choose $\bar{A}=\left\langle A_{\delta}: \delta \in S^{*}\right\rangle$ by induction on $\delta$, each time choosing $A_{\delta} \in \mathcal{X}_{\delta}$ so as to have a finite intersection with $\operatorname{Inv}_{\bar{B}_{i}^{*}}\left(x, C_{\delta}\right)$ for all $x \in \bigcup_{i<i^{*}} B_{i}^{*}$ and to be distinct from $A_{\beta}$ for $\beta<\delta$. This is possible because each $\operatorname{Inv}_{\bar{B}_{i}^{*}}\left(x, C_{\delta}\right)$ is a countable subset of $C_{\delta}$ and hence intersects at most $2^{\aleph_{0}}<\mu$ elements of $\mathcal{X}_{\delta}$, and there are at most $\lambda \cdot\left|i^{*}\right|<\mu^{\aleph_{0}}$ subsets of $C_{\delta}$ used as invariants.

Having chosen $\bar{A}$ consider $B[\bar{A}]$ with its associated filtration $\bar{B}[\bar{A}]$, and suppose that $f: B[\bar{A}] \rightarrow B_{i}^{*}$ is an embedding which preserves " $\in \bigcup_{n<\omega} B_{n}$ ", for some $i<i^{*}$. Let $E$ be a club of $\lambda$ such that $\delta \in E \Longrightarrow f^{\prime \prime} B_{\delta}[\bar{A}]=f " B[\bar{A}] \cap B_{i}^{*, \delta}$, and let $\delta \in S^{*}$ be such that $C_{\delta} \subseteq E$. Let $\eta_{\delta}: \omega \rightarrow A_{\delta}$ be the increasing enumeration of $A_{\delta}$. Hence for $n<\omega$, we have $\eta_{\delta}(n) \in \operatorname{Inv}_{\bar{B}[\bar{A}]}\left(b_{\delta}^{0}, C_{\delta}\right)$ by virtue of $n$, as exemplified by say $c_{n}^{\delta}$. Let $m_{n}$ for $n<\omega$ be such that $f\left(c_{n}^{\delta}\right) \in B_{m_{n}}\left(B_{i}^{*}\right)$. Note that $f\left(c_{n}^{\delta}\right) \in$ $B_{i}^{*, \min \left(C_{\delta} \backslash\left(\eta_{\delta}(n)+1\right)\right.} \backslash B_{i}^{*, \eta_{\delta}(n)}$ by the choice of $E$.

Now notice that for $n_{1} \neq n_{2}$ we have $m_{n_{1}} \neq m_{n_{2}}$, as $f\left(c_{\delta}^{n_{l}}\right) \geq f\left(b_{\delta}^{0}\right) \neq 0$ and $f\left(c_{\delta}^{n_{l}}\right) \in B_{m_{n_{l}}}\left(B_{i}^{*}\right)$, for $l \in 1,2$. By the choice of $E$ and $C_{\delta}$, we have that whenever $m_{n} \neq 0$, the ordinal $\eta(n)$ is $\operatorname{in}_{\operatorname{Inv}_{\bar{B}_{i}^{*}}}\left(f\left(b_{\delta}^{0}\right), C_{\delta}\right)$, by virtue of $m_{n}$. Hence $\operatorname{Inv}_{\bar{B}_{i}^{*}}\left(f\left(b_{\delta}^{0}\right), C_{\delta}\right) \cap A_{\delta} \supseteq\left\{\eta(n): m_{n} \neq 0\right\}$ which is infinite, contradicting the choice of $\bar{A}$.

\section{A POSitive CONSISTENCY RESUlt}

Theorem 3.1. Suppose that $\lambda^{<\lambda}=\lambda>\aleph_{0}$ in a model $V$ of (a sufficient amount of) $Z F C$. Then there is a cardinality and cofinality preserving extension $V[G]$ of $V$ in which $2^{\lambda^{+}}>\lambda^{++}$and in which there are $\lambda^{++}$c-algebras of size $\lambda^{+}$which are jointly c-universal for c-algebras of size $\lambda^{+}$.

Consequently, in $V[G]$ there is a UEC of weight $\lambda^{++}$which is universal for UEC of weight $\lambda^{+}$and in fact there are $\lambda^{++}$UEC of weight $\lambda^{+}$which are jointly universal for UEC of size $\lambda^{+}$(while $2^{\lambda^{+}}>\lambda^{++}$holds). 
Proof. The main theorem of [DžSh04 is (see also She93]) that under the above assumptions there is a cardinality and cofinality preserving extension $V[G]$ of $V$ in which $2^{\lambda^{+}}>\lambda^{++}$and in which every abstract elementary class $\mathcal{K}$ with JEP and AP that has a workable $\lambda$-approximation family $\lambda$-approximating it admits $\lambda^{++}$ elements of size $\lambda^{+}$which are jointly universal for $\mathcal{K}_{\lambda^{+}}$. It hence suffices to show that the class $\mathcal{K}$ of c-algebras under c-embeddings is such a class in the extension $V[G]$ guaranteed by DžSh04]. This consists of checking a number of required axioms, whose statements as well as terms used in the above are given in \$1. The definitions given there are a special case of those in DžSh04 because we only need to use the quoted special case of the main theorem of that paper. At the end, the second part of the theorem will follow by Bell's Theorem 1.5.

Let $\mathcal{M}_{\lambda}^{\text {ap }}$ be the set of models $M$ of $\tau=\left\{B_{n}: n<\omega\right\} \cup\{\wedge, \vee, 0,1, \leq\}$ whose domain is a set $u_{M} \in\left[\lambda^{+}\right]^{<\lambda}$, where each $B_{n}$ is a unary relational symbol, $\wedge$ and $\vee$ are binary function symbols and 0,1 are constants with $0_{M}=0,1_{M}=1$, and such that $M$ satisfes the axioms for a c-algebra with $B_{n}(M)=\left(B_{n}\right)^{M}$ We also allow $M=\emptyset$.

We order $\mathcal{M}_{\lambda}^{\text {ap }}$ by letting $M \leq_{\text {ap }} N$ iff $M$ is a (possibly empty) submodel of $N$ and for all $r \in M$ and a finite sequence $\left\langle n_{0}, \ldots, n_{k}\right\rangle$ in $\omega$,

$$
N \vDash "\left(\bigwedge_{i \leq k} \exists q_{i} \in B_{n_{i}}\right)\left[r \leq \bigvee_{i \leq k} q_{i}\right] " \Longrightarrow M \vDash "\left(\bigwedge_{i \leq k} \exists q_{i} \in B_{n_{i}}\right)\left[r \leq \bigvee_{i \leq k} q_{i}\right] " .
$$

Finally we let $\mathcal{K}_{\lambda}^{\text {ap }}$ be the set of all $M \in \mathcal{M}_{\lambda}^{\text {ap }}$ that satisfy that for every $\delta$ divisible by $\lambda$ we have $M\left\lceil\delta \leq_{\text {ap }} M\right.$. Firstly we check that $\mathcal{K}_{\lambda}^{\text {ap }}$ is a workable $\lambda$-approximation family. The axioms (a)-(c) are obviously satisfied and (d) is easily seen to hold. To check the end extension/amalgamation axiom we shall use the following claims.

Claim 3.2. Suppose that $\emptyset \neq M_{0} \leq_{\text {ap }} M_{1}$ in $\mathcal{K}_{\lambda}^{\text {ap }}$. Then for every $n<\omega$ and $p \in B_{n}\left(M_{1}\right) \backslash B_{n}\left(M_{0}\right)$ and $r \in M_{0}$ we have $r \leq p \Longrightarrow r=0$.

Proof of the Claim. Suppose otherwise, and let $r$ exemplify that. Then $M_{1} \vDash$ " $\left(\exists q \in B_{n}\right)[r \leq q]$ ", as this is exemplified by $p$, so $M_{0}$ satisfies the same by the definition of $\leq_{\text {ap }}$. Let $q$ exemplify this. Then in $M_{1}$ we have that $p$ and $q$ are distinct elements of $B_{n}$ with $p \wedge q \geq r$, a contradiction.

Claim 3.3. Suppose that $M_{1}$ and $M_{2}$ are elements of $\mathcal{K}_{\lambda}^{\text {ap }}$ such that $M_{1} \cap M_{2}$ is an element $M_{0}$ of $\mathcal{K}_{\lambda}^{\text {ap }}$ and $M_{0} \leq_{\text {ap }} M_{1}, M_{2}$. Then there is a c-algebra $B\left[M_{1}, M_{2}\right]$ generated by $\bigcup_{n<\omega}\left[B_{n}\left(M_{1}\right) \cup B_{n}\left(M_{2}\right)\right]$ freely except for the equations holding in $M_{1}$ and $M_{2}$ and

$(*)_{n}$

$$
p \wedge q=0\left(p \neq q \in B_{n}\left(M_{1}\right) \cup B_{n}\left(M_{2}\right)\right)
$$

for $n<\omega$, that c-embeds both $M_{1}$ and $M_{2}$ as c-subalgebras. Moreover, there is an isomorphic copy $M$ of $B\left[M_{1}, M_{2}\right]$ which is an element of $\mathcal{K}_{\lambda}^{\text {ap }}$ with the property that $M_{1}, M_{2} \leq_{\text {ap }} M$ and such that for any $\delta$ divisible by $\lambda$ we have that $M \uparrow \delta$ is generated by $\left(\bigcup_{n<\omega}\left[B_{n}\left(M_{1}\right) \cup B_{n}\left(M_{2}\right)\right]\right) \cap \delta$.

Proof of the Claim. Let us assume that $M_{0} \neq \emptyset$ as otherwise the situation is similar but easier. First note that for $n \neq m$ we have

$$
\left[B_{n}\left(M_{1}\right) \cup B_{n}\left(M_{2}\right)\right] \cap\left[B_{m}\left(M_{1}\right) \cup B_{m}\left(M_{2}\right)\right]=\emptyset
$$

\footnotetext{
${ }^{2}$ Note that this definition with $\lambda=\aleph_{0}$ yields $\mathcal{M}_{\lambda}^{\text {ap }}=\{\emptyset\}$, which is why in Theorem 3.1 we need $\lambda>\aleph_{0}$ even though the theorem from DžSh04 remains valid for $\lambda=\aleph_{0}$. Also note that the definition of $\mathcal{M}_{\lambda}^{\text {ap }}$ is not first order.
} 
by the fact that $M_{0} \leq$ ap $M_{1}, M_{2}$ and $M_{0}=M_{1} \cap M_{2}$. So if we verify that there are no contradictions in requiring $\bigwedge_{n<\omega}(*)_{n}$, we can define a Boolean algebra $B\left[M_{1}, M_{2}\right]$ which has $M_{1}$ and $M_{2}$ as subalgebras and is generated by $\bigcup_{n<\omega}\left[B_{n}\left(M_{1}\right) \cup B_{n}\left(M_{2}\right)\right]$ freely except for $\bigwedge_{n<\omega}(*)_{n}$, and if this algebra ends up being a c-algebra, then both $M_{1}$ and $M_{2}$ will be c-embedded into it. For the former statement to hold, we need to verify that for every $n$ and $p \in B_{n}\left(M_{1}\right) \backslash B_{n}\left(M_{2}\right), q \in B_{n}\left(M_{2}\right) \backslash B_{n}\left(M_{1}\right)$ we can require that $p \wedge q=0$. This attempt may fail if there is $r \in M_{0} \backslash\{0\}$ such that $M_{1} \vDash r \leq p$ and $M_{2} \vDash r \leq q$. But such an $r$ cannot exist by Claim 3.2 .

Now to verify that $B\left[M_{1}, M_{2}\right]$ is a c-algebra, we need to know that there is no finite $F \subseteq \bigcup_{n<\omega} B_{n}\left(M_{1}\right) \cup B_{n}\left(M_{2}\right)$ with $\bigvee F=1$. Suppose $F$ is such and let $F^{\prime}=F \cap\left(M_{1} \backslash M_{2}\right)$ and $F^{\prime \prime}=F \backslash F^{\prime}$. Note that $F^{\prime}, F^{\prime \prime} \neq \emptyset$. Let $p=\bigvee F^{\prime}$, so $p \in M_{1}$, and let $q=\bigvee F^{\prime \prime}$, so $q \in M_{2}$. In $B\left[M_{1}, M_{2}\right]$ we have $p \vee q=1$, so as this Boolean algebra is generated freely over $M_{1}$ and $M_{2}$, we must have that $q \in M_{0}$ and $p \vee q=1$.

Let $\left\langle n_{0}, \ldots, n_{k}\right\rangle$ be some finite sequence of natural numbers possibly with repetitions such that for every element $r$ of $F^{\prime}$ we have $r \in B_{n_{i}}\left(M_{1}\right)$ for some $i$. Using that $M_{0} \leq_{\text {ap }} M_{1}$ and considering $-q \in M_{0}$ we obtain that the following holds in $M_{0}$ :

$$
\left(\bigwedge_{i \leq k} \exists r_{i} \in B_{n_{i}}\left(M_{0}\right)\left[\bigvee_{i \leq k} r_{i} \vee q=1\right] .\right.
$$

Let $q_{0}, \ldots, q_{m} \in \bigcup_{n<\omega} B_{n}\left(M_{2}\right)$ be such that $\bigvee_{j \leq m} q_{j}=q$. By $M_{0} \leq_{\text {ap }} M_{2}$ we may find $s_{0}, \ldots, s_{m} \in \bigcup_{n<\omega} B_{n}\left(M_{2}\right)$ such that $q \leq \bar{\bigvee}_{j \leq m} s_{j}$. But then in $M_{0}$ we have $\bigvee_{i \leq k} r_{i} \vee \bigvee_{j \leq m} s_{j}=1$, a contradiction.

As for the last requirement on $M$, since $M_{1}$ and $M_{2}$ are both of size $<\lambda$ and since they both are in $\mathcal{K}_{\text {ap }}$, we may choose the new elements of $M$ so that for any $\delta$ divisible by $\lambda$ we have that $M \uparrow \delta$ is generated by $\left(\bigcup_{n<\omega}\left[B_{n}\left(M_{1}\right) \cup B_{n}\left(M_{2}\right)\right]\right)$ $\cap \delta$. We now need to verify that $M$ is in $\mathcal{K}_{\lambda}^{\text {ap }}$ by checking that for every such $\delta$ we have $M\left\lceil\delta \leq_{\text {ap }} M\right.$. This follows from the requirement on $M_{1}$ and $M_{2}$ since $B_{n}(M)=B_{n}\left(M_{1}\right) \cup B_{n}\left(M_{2}\right)$ for all $n$. Finally to see that $M_{1}, M_{2} \leq_{\text {ap }} M$ we use an argument similar to the one showing that $M$ is a c-algebra.

Let $\delta, M_{0}, M_{1}, M_{2}$ be as in the end extension/amalgamation axiom. We define $M_{3}$ to be $M$ from Claim 3.3 . For the second clause of the axiom we simply take $M_{3}$ to be the closure of $M_{1} \cup M_{2}$ in $M$.

The local cardinality axiom follows from the assumption $\lambda^{<\lambda}=\lambda$. The uniformity axiom is easily checked, and the density axiom follows by adding an extra generator to $B_{0}(M)$ for a given $M \in \mathcal{K}_{\lambda}^{\text {ap }}$. For the amalgamation axiom we first need to observe that when we are given $M_{0}, M_{1}$ and $M_{2}$ as in the assumptions, we can by using a lawful isomorphism if necessary assume that $M_{1} \cap M_{2}=M_{0}$. Then we can continue as in the proof of the end extension/amalgamation axiom. It remains to check the workability, which is the main point of the proof.

Let us suppose that we are in the situation of the assumptions of Definition 1.8. Let $N_{0} \stackrel{\text { def }}{=}\left(N_{1}\left\lceil\delta_{1}\right)\right.$ and $M_{0} \stackrel{\text { def }}{=}\left(M_{1} \uparrow \delta_{1}\right)$. We first choose $M \in \Gamma$ so that $M_{1}, M_{2} \leq$ ap $M$, which can be done by the directedness of $\Gamma$. Consequently, $|M| \subseteq$ Ev. Our task is to define $N$ as required. We shall proceed similarly to the proof of Claim 3.3. first defining a c-algebra, let us denote it by $B=B\left[N_{1}, N_{2}, M\right]$, and then taking an isomorphic copy of it as $N$. Let $B_{n}(B) \stackrel{\text { def }}{=} \bigcup_{n<\omega}\left[B_{n}\left(N_{1}\right) \cup\right.$ $B_{n}\left(N_{2}\right) \cup B_{n}(M)$ ]. We wish to define $B$ as a Boolean algebra to be generated by 
$\bigcup_{n<\omega} B_{n}$ freely except for the equations of $M, N_{1}$ and $N_{2}$ and

$(* *)_{n}$

$$
p \wedge q=0\left(p \neq q \in B_{n}(B)\right)
$$

for $n<\omega$. As in the proof of Claim 3.3 we shall assume that all the models in question are non-empty, as the opposite situation is in fact easier to handle. We first note that for $n \neq m<\omega$ we have $B_{n}(B) \cap B_{m}(B)=\emptyset$ by the fact that $N_{l}\left\lceil\mathrm{Ev}=M_{l}\right.$ and $M_{l} \leq_{\text {ap }} M$ for $l=\{1,2\}$, while $N_{1} \cap N_{2}=N_{0}$. Now suppose that there is a contradiction among the equations of $(* *)_{n}$, so for some $n<\omega$ there are $p \neq q \in B_{n}(B)$ such that $p \wedge q=0$ is contradicted by the requirement that $N_{1}, N_{2}$ and $M$ are embedded into $B$ as subalgebras. Clearly, it cannot be the case that $p, q$ come from any one among $N_{1}, N_{2}$ and $M$. We discuss the remaining possibilities.

Case 1. $p \in N_{1} \backslash N_{2}$ and $q \in N_{2} \backslash N_{1}$, or vice versa. We without loss of generality assume that $p \in N_{1}$ and $q \in N_{2}$. Hence there is $r \neq 0$ in $N_{0}$ such that $N_{1} \vDash$ " $r \leq p$ " and $N_{2} \vDash$ " $r \leq q$ ". Since $N_{0} \leq$ ap $N_{1}$, by Claim 3.2 we have that $r=0$.

Case 2. $p \in N_{l} \backslash M$ for some $l \in\{1,2\}$ and $q \in M \backslash\left(M_{1} \cup M_{2}\right)$, or vice versa. Without loss of generality assume that $p \in N_{l}$ and $q \in M$. We might have a problem only if for some $r \in M_{0} \backslash\{0\}$ we have $r \leq p$ and $r \leq q$, which would contradict Claim 3.2 ,

It follows that $B$ is a well-defined Boolean algebra that embeds $N_{1}, N_{2}$ and $M$. To verify that it is a c-algebra, suppose that $F \subseteq \bigcup_{n<\omega} B_{n}(B)$ is finite and $\bigvee F=1$. Let $F_{0}=F \cap M$ and $F_{l}=F \cap\left(N_{l} \backslash M\right)$, for $l \in\{1,2\}$, and for $m<3$ let $a_{m}=\bigvee F_{m}$. It is clear that at least two among $F_{0}, F_{1}, F_{2}$ must be non-empty. Let us discuss the possible ways this can happen. The main case is Case (a), the proofs of the other cases are a derivation of the proof of Case (a).

Case (a). None among $\left\{F_{0}, F_{1}, F_{2}\right\}$ is empty. In $N$ we have $\bigvee_{l<3} a_{l}=1$, so $a_{1} \overline{\vee a_{2} \geq-} a_{0}$. As $B$ is generated freely except for the requirements that it embeds $N_{1}, N_{2}$ and $M$, and $\bigwedge_{n<\omega}(* *)_{n}$, there must be $r \in\left(N_{1} \cup N_{2}\right) \cap M=M_{1} \cup M_{2}$ such that $r \geq-a_{0}$ holds in $M$, while $a_{1} \vee a_{2} \geq r$ holds in $N$.

Subcase (a1). $r \in M_{2}$. As $M_{2} \leq_{\text {ap }} M$ and $-r \leq a_{0}$, we can find $a_{0}^{\prime} \in M_{2}$ which is a finite disjunction of elements of $\bigcup_{n<\omega} B_{n}\left(M_{2}\right)$ and such that $-r \leq a_{0}^{\prime}$ holds in $M_{2}$. Hence $a_{1} \vee a_{2} \vee a_{0}^{\prime}=1$ holds in $N$, and so $a_{1} \geq-\left(a_{2} \vee a_{0}^{\prime}\right)$. Using again the fact that $N$ is generated freely except for the specified equations, there must be $q \in N_{1} \cap N_{2}=N_{0}$ such that $a_{1} \geq q$ holds in $N_{1}$, while $-\left(a_{2} \vee a_{0}^{\prime}\right) \leq q$ holds in $N_{2}$. Since $N_{0} \leq$ ap $N_{1}$ there must be $b_{1} \in N_{0}$ which is a finite disjunction of elements in $\bigcup_{n<\omega} B_{n}\left(N_{0}\right)$ such that $q \leq b_{1}$. Then $a_{0}^{\prime} \vee b_{1} \vee a_{2}=1$ holds in $N_{2}$, a contradiction. Subcase (a2). $r \in M_{1}$. Analogous to Subcase (a1).

$\overline{\text { Case (b). } \quad F_{0}}=\emptyset$. We have $a_{1} \vee a_{2}=1$, so $a_{1} \geq-a_{2}$ holds in $N$. By the fact that $N$ is generated freely except for the specified equations, there must be $r \in N_{1} \cap N_{2}=N_{0}$ such that $a_{1} \geq r$ holds in $N_{1}$ and $r \geq-a_{2}$ holds in $N_{2}$. Using $N_{0} \leq_{\text {ap }} N_{1}$ we find $a_{1}^{\prime}$ which is a finite disjunction of elements of $\bigcup_{n<\omega} B_{n}\left(N_{0}\right)$ and that satisfies $a_{1}^{\prime} \geq r$ in $N_{0}$. Then $a_{1}^{\prime} \vee a_{2}=1$ in $N_{2}$, a contradiction.

Case (c). $\quad F_{1}=\emptyset$. We have $a_{0} \vee a_{2}=1$, so $a_{0} \geq-a_{2}$ holds in $N$. By the fact that $N$ is generated freely except for the specified equations, there must be $r \in M \cap N_{2}=M_{2}$ such that $a_{0} \geq r$ holds in $M$ and $r \geq-a_{2}$ holds in $N_{2}$. Now proceed as in Case (b).

Case (d). $F_{2}=\emptyset$. Analogous to case (c). 
Now that we have obtained $B$ we can as in Claim 3.3 get an isomorphic copy $M$ which is as required. This finishes the proof of workability.

Now we need to prove that $\mathcal{K}$ is an abstract elementary class with JEP and AP. Let $\tau$ be the same vocabulary as that of $\mathcal{K}_{\lambda}^{\text {ap }}$. Most of the requirements are obviously satisfied. If $\bar{M}$ is a continuous chain of c-algebras which are c-embedded into each other, clearly the union of the chain is the lub. We have $L S(\mathcal{K})=\aleph_{0}$. To check JEP and AP use the method of the proof of Claim 3.3 .

Finally we need to check that $\mathcal{K}_{\lambda}^{\text {ap }} \lambda$-approximates $\mathcal{K}$. It is obvious that $\mathcal{K}_{\lambda}^{\text {ap }}$ tends to strongly $\lambda$-approximate $\mathcal{K}$. Suppose $M^{*} \in \mathcal{K}_{\lambda^{+}}$, and without loss of generality $\left|M^{*}\right| \subseteq \lambda^{+} \cap$ Ev. By reenumerating, we can assume that for every $\delta$ divisible by $\lambda$ we have $M^{*} \mid \delta \prec M^{*}$. Let $\Gamma \stackrel{\text { def }}{=}\left\{M: M \prec M^{*} \&\|M\|<\lambda\right\}$. Now it clearly follows that $\Gamma \in K_{\mathrm{md}}^{-}\left[\mathcal{K}_{\mathrm{ap}}^{\lambda}\right]$ and $M_{\Gamma}$ is isomorphic to $M^{*}$.

\section{REFERENCES}

[Bel00] M. Bell. Universal uniform Eberlein compact spaces. Proceedings of AMS, 128, 7:21912197, 2000. MR:1676311 (2000m:54019)

[YRM77] Y.Benyamini, M. E. Rudin, and M.Wage. Continuous images of weakly compact subsets of Banach spaces. Pacific Journal of Mathematics, 70:309-324, 1977. MR0625889 $(58: 30065)$

[Dža98] M. Džamonja. On uniform Eberlein compacta and c-algebras. Topology Proceedings, 23:143-150, 1998. MR1743805 (2001f:06024)

[DžSh04] M. Džamonja and S. Shelah. On the existence of universal models. Archive for Mathematical Logic, 43:901-936, 2004. MR2096141

[She94] S. Shelah. Cardinal Arithmetic. Oxford University Press, 1994. MR:1318912 (96e:03001)

[She93] S. Shelah. The universality spectrum: Consistency for more classes. In Combinatorics, Paul Erdös is Eighty, Bolyai Society Mathematical Studies, 1:403-420, 1993. MR.1249724 (95a:03043)

School of Mathematics, University of East Anglia, Norwich, NR4 7TJ, United KingDOM

E-mail address: h020@uea.ac.uk 